\title{
Stability of Mobile Slotted ALOHA Network with Rayleigh Fading, Shadowing, and Near-Far Effect
}

\author{
CORNELIS VAN DER PLAS AND JEAN-PAUL M. G. LINNARTZ, MEMBER, IEEE
}

\begin{abstract}
Queueing of packets in a mobile slotted ALOHA network with Rayleigh fading, shadowing, and UHF groundwave propagation is studied, using a finite Markov chain model. Receiver capture, assisted by all three propagation mechanisms, reduces bistability of the network substantially, compared to wired networks: the stability of mobile networks is less sensitive to the retransmission strategy. The near-far effect is highlighted, since the total network performance and the spatial distribution of the attempted traffic interact.
\end{abstract}

\section{INTRODUCTION}

QLOTTED ALOHA [1], [2] is a well-known multiple$\checkmark$ access protocol in mobile networks. Mobile terminals transmit packets over a common radio channel to a central base station over a common radio channel in predefined time slots, but without any mutual control or centralized regulation except for positive acknowledgments of reception. If two or more terminals transmit simultaneously, a packet collision occurs; this mutual interference may result in loss of packets. Such packets are retransmitted after waiting a random number of time slots after the missing acknowledgment from the base station. In most cable networks, e.g. local area networks (LAN's), any collision may be assumed to destroy all packets involved. This limits the channel capacity of slotted ALOHA to $37 \%$, and the network exhibits a tendency to instability [2]. Colliding packets from mobile terminals, on the other hand, arrive with different power levels. Since the strongest packet may be able to capture a discriminating receiver, enhanced capacity is obtained [3]-[10], [25].

In the present paper, dynamic network behavior is investigated, using the classical ALOHA queueing model [12], [13] as extended in [11] to mobile channels (Section II). We focus on the assessment of receiver capture probabilities, based on a general propagation model for mobile radio communications described in Section III. In Section IV, a spatial weighing method is developed which takes Rayleigh fading, shadowing, and the near-far effect into account analytically. Section V compares the effects of the three mechanisms on

Manuscript received December 12, 1989; revised May 30, 1990.

C. van der Plas was with the Telecommunications and Traffic-Control Systems Group, Delft University of Technology, Delft, the Netherlands. He is now with Intercai B.V., P.O. Box 4187, 3006 AD Rotterdam, the Netherlands.

J. P. M. G. Linnartz is with the Telecommunications and Traffic-Control Systems Group, Delft University of Technology, P.O. Box 5031, 2600 GA Delft, the Netherlands.

IEEE Log Number 9038887
TERMINAL MODEL

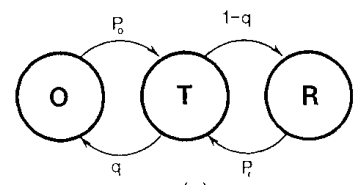

(a)

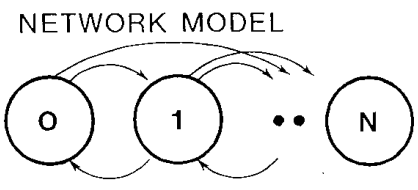

(b)

Fig. 1. Markov chain model for (a) the individual terminals and (b) the entire network.

network stability. The near-far effect is investigated in Section VI; since the majority of all retransmissions originate from (distant) areas with poor propagation [8], [9], we compare uniform and nonuniform spatial distributions of traffic.

\section{Queuing Model}

The slotted ALOHA network is assumed to comprise $N$ identical, independently operating mobile terminals. The behavior of each terminal is described by a finite Markov chain with three states [11]-[13] (Fig. 1(a)). In the origination state $(O)$, a packet is generated (and transmitted in the next time slot) with probability $p_{0}$. In the transmission state $(T)$, the terminal is busy with either transmitting a new packet or retransmitting a previously collided packet. The mobile terminal returns to the $O$ state if it receives a positive acknowledgment at the end of the time slot during which a packet is transmitted; otherwise it enters the retransmission or backlogged state $(R)$. From the latter state, retransmission (i.e., transition into the $T$ state) occurs with probability $p_{r}$. A backlogged terminal is blocked in the sense that no new messages arrive when the terminal is in state $R$.

The behavior of the entire slotted ALOHA network is also modeled by means of a finite Markov chain (Fig. 1(b)). With $N$ terminals, we have $N+1$ states, defined as the number of terminals in state $R$ at the start of a time slot. During a time slot, $i$ packets are transmitted whenever $i$ terminals, each being in an $O$ or $R$ state, change into the $T$ state at the same time. The probability $q_{i}$ that one out of the $i$ colliding 
packets is successfully received (captured) is studied in Section IV: Namislo [11] expressed the state transition probabilities for a network with capture in terms of the number of participating terminals $N$ and the probabilities of generation $p_{0}$, capture $q_{i}$ and retransmission $p_{r}$. State probabilities $\pi_{n}$ can then be obtained recursively [2]. Further, the throughput $S_{n}$ in state $n$ is found from the probability to successfully transmit a packet in a time slot and is determined by averaging the probability of $i(i=j+k)$ colliding packets, given a backlog of $n$ terminals, i.e.,

$$
\begin{aligned}
S_{n}=\sum_{k=0}^{N-n}\left(\begin{array}{c}
N-n \\
N-n-k
\end{array}\right) p_{0}^{k}\left(1-p_{0}\right)^{N-n-k} \\
\cdot \sum_{j=0}^{n}\left(\begin{array}{l}
n \\
j
\end{array}\right) p_{r}^{j}\left(1-p_{r}\right)^{n-j} q_{j+k} .
\end{aligned}
$$

Stability depends on the expected drift $d_{n}$ in each state, defined as the difference between the expected input and the expected output traffic in that state, i.e.,

$$
d_{n} \triangleq(N-n) p_{0}-S_{n} \quad \text { (states per time slot) }
$$

In a simulation to be described in Section VI, this drift is obtained directly as the average motion toward another (higher or lower) state, when the network is in state $n$. The network is expected to operate in a state near an equilibrium point, i.e., where the expected drift (2) crosses zero with negative derivative [11], [12].

The average total network throughput $S$ is found by averaging (1) over the state probabilities $\pi_{n}$, viz.,

$$
S=\sum_{n=0}^{N} \pi_{n} S_{n} \quad \text { (packets per time slot) }
$$

In a well-functioning network in equilibrium, the channel throughput $S$ must equal the newly generated traffic, so

$$
S=\left(N-\pi_{S}\right) p_{0},
$$

with $\pi_{S}$ the average backlog, defined as the average network state

$$
\pi_{S} \triangleq \sum_{n=0}^{N} \pi_{n} n
$$

The expected delay $D$ is

$$
D=\frac{\pi_{S}}{S} \quad \text { (time slots) }
$$

\section{Propagation Model}

The mobile radio channel can be characterized statistically by three independent, multiplicative, propagation mechanisms, namely multipath fading, shadowing and UHF groundwave propagation [16]. The latter mechanism gives rise to the near-far effect and determines the area-mean power $p_{a}$, i.e., the received power averaged over some area in order to eliminate the former two local mechanisms. The normalized area-mean power received from a mobile terminal at a distance $r$ from the base station is taken to have the form

$$
p_{a}=r^{-\beta},
$$

with the empirical constant $\beta$ in the range of three to four. Shadowing is assumed superimposed on the near-far effect (7). This (slow) statistical fluctuation is described by a lognormal distribution of the local-mean power $p_{1}$ about the area-mean power $p_{a}$, i.e., if the local-mean power is expressed in nepers, it has a normal distribution about the area mean, with a logarithmic standard deviation $\sigma_{s}$. Finally, multipath reception causes Rayleigh fading. Thus the instantaneous received power $p_{s}$ of a signal from a mobile terminal is exponentially distributed about the local-mean power, and has a faster fluctuation.

Combining all three propagation mechanisms, the unconditional probability density function (pdf) of the instantaneous power $p_{s}$ of a received packet is [17]

$$
\begin{aligned}
f_{p_{s}}\left(p_{s}\right)=\int_{0}^{\infty} \int_{0}^{\infty} \frac{1}{p_{1}} \exp \left(-\frac{p_{s}}{p_{1}}\right) \frac{f(r)}{\sqrt{2 \pi} \sigma_{s} p_{1}} \\
\cdot \exp \left\{-\frac{\left(\ln p_{1}+\beta \ln r\right)^{2}}{2 \sigma_{s}^{2}}\right\} d r d p_{1} .
\end{aligned}
$$

Here, $f(r)$ is the pdf of the propagation distance describing the spatial distribution of the offered packet traffic around the central receiver [4]. The received packet powers $p_{s}$ from two successive transmissions by the same terminal are assumed entirely uncorrelated because of Rayleigh fading and shadowing.

\section{Capture Model}

Conditional capture probabilities as required in the queueing model (1) are obtained for the propagation scenario (8). A test packet is assumed to capture the base station if and only if its instantaneous power exceeds the instantaneous joint interference power by at least a threshold factor $z$ [4], [7]-[9], [25], which reflects the capture performance of the applied receiver, modulation and coding techniques. During the capture of a packet, the received (instantaneous) power $p_{s}$ is assumed constant [18]. The ALOHA channel is principally contention limited, so thermal and man-made noise are neglected, as is the influence of the irreducible errors due to random Doppler modulation.

For this type of Rayleigh fading channels, the weight function approach [8], [9], based on Laplace transforms [19], yields a convenient method of analyzing capture probabilities. This method applies the Laplace image $\phi_{P n}(\cdot)$ of the pdf of the instantaneous joint interference power $p_{n}$, defined as

$$
\phi_{P n}(v) \triangleq \int_{0}^{\infty} \exp (-v x) f_{P n}(x) d x .
$$

The probability of capture, given the local mean power of the desired test packet $p_{1 t}$, can be expressed as [8], [9], [20]

$$
\begin{aligned}
& \operatorname{Pr}\left(\text { capture } \mid i-1, p_{1 t}\right) \\
& \quad=\operatorname{Pr}\left(p_{s} / p_{n}>z \mid i-1, p_{1 t}\right) \\
& \quad=\int_{z}^{\infty} \int_{0}^{\infty} \frac{1}{p_{1 t}} \exp \left\{-\frac{x y}{p_{1 t}}\right\} f_{P_{n}}(x) \times d x d y
\end{aligned}
$$




$$
\begin{aligned}
& =\int_{0}^{\infty} \exp \left\{-\frac{x z}{p_{1 t}}\right\} f_{P n}(x) d x \\
& \triangleq \phi_{P n}\left(z / p_{1 t}\right) .
\end{aligned}
$$

If the interference power $p_{n}$ is due to incoherent accumulation of $i-1$ independently fading signals, the joint pdf is the ( $i-1$ )-fold convolution of the pdf of the individual signal powers. Laplace transformation results in the $(i-1)$ th power of the characteristic function of the pdf of one single interferer, viz.

$$
\operatorname{Pr}\left(\text { capture } \mid i-1, p_{1 t}\right)=\phi_{P s}^{i-1}\left(z / p_{1 t}\right) .
$$

Rayleigh fading of the test signal is included in (10) since this probability is conditional on the local mean power $p_{1 t}$ received from the mobile transmitter. The Laplace image $\phi_{P S}(v)$ of (8) is expressed as

$$
\begin{aligned}
\phi_{P S}(v)=\int_{0}^{\infty} \int_{0}^{\infty} \frac{1}{1+v p_{1}} \cdot \frac{f(r)}{\sqrt{2 \pi} \sigma_{s} p_{1}} \\
\cdot \exp \left(-\frac{\left(\ln p_{1}+\beta \ln r\right)^{2}}{2 \sigma_{s}^{2}}\right) d r d p_{1} .
\end{aligned}
$$

The integral transform (11) of the spatial distribution can be interpreted as a weighing of the vulnerability of the test packet to interference at distance $r$, i.e.,

$$
\phi_{P s}(v)=\int_{0}^{\infty} W(v, r) f(r) d r,
$$

where the vulnerability weight function $W(\cdot)(0<W<1)$ is defined by

$$
W(v, r) \triangleq \frac{1}{\sqrt{\pi}} \int_{-\infty}^{\infty} \frac{\exp \left(-x^{2}\right)}{1+v r^{-\beta} \exp \{x \sigma \sqrt{2}\}} d x .
$$

For channels without shadowing $\left(\sigma \rightarrow 0\right.$, and thus $p_{1}=r^{-\beta}$ ), the simpler weight function discovered in [8], [9]

$$
W(v, r)=\frac{1}{1+v r^{-\beta}}
$$

is recovered, and the conditional probability of capture becomes

$\operatorname{Pr}\left\{\right.$ capture $\left.\mid i-1, r_{t}\right\}=\left[\int_{0}^{\infty} \frac{f(r)}{1+z r_{t}^{\beta} r^{-\beta}} d r\right]^{i-1}$.

Here, $r_{t}$ is the distance between the base station and the terminal transmitting the test signal. Fig. 2 illustrates that the capture model proposed in [3] is recovered by replacing (13) by a step function. The latter model [3], [6], [26] ignores cumulation of interference power, Rayleigh fading and shadowing: it assumes capture if and only if no colliding packet is transmitted within the "vulnerability circle" with radius $r_{t}$ - $\sqrt[\beta]{z}$ around the central receiver. However, active terminals outside this circle do introduce some interference. Accordingly, the integral in (12) has its upper limit at infinity. Apparently, Rayleigh fading and shadowing of the interfering packet traffic change the circle adopted by Abramson into the

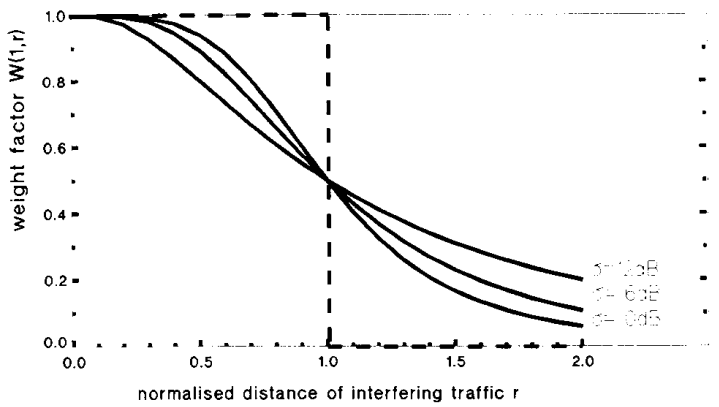

Fig. 2. Weight factor $W(1, r)(-)$ and its approximation (- $)$ [3], describing the vulnerability of a test packet to interference at normalized distance $r$. Shadowing is 0,6 and $12 \mathrm{~dB}$.

softer transition region described by the vulnerability weight function (13). A further comparison of the capture criteria in the models suggested in [1] and [4] is presented in [25].

In order to investigate the network in its entirety from the Markov chain model (1), capture probabilities unconditional on the location of the mobile transmitting the test packet are required. To this end, probability (10) or (12) must be averaged over the pdf due to the shadowing and near-far effects on the test signal. The capture probability, given that $i-1$ packets interfere, then becomes

$$
\begin{aligned}
& \operatorname{Pr}\left\{\text { capture }\{i-1\}=\int_{0}^{\infty} \int_{0}^{\infty} \frac{f\left(r_{t}\right)}{\sqrt{2 \pi} \sigma_{s} p_{1 t}}\right. \\
& \cdot \exp \left\{\frac{-\left(\ln p_{1 t}+\beta \ln r_{t}\right)^{2}}{2 \sigma_{s}^{2}}\right\}\left[\phi_{P_{s}}\left(z / p_{1 t}\right)\right]^{i-1} d p_{1 t} d r_{t} .
\end{aligned}
$$

Despite our assumption of a discrete number of $N$ participating terminals in the queueing model, the packet traffic is taken to be continuously spread over the coverage area for the assessment of the capture probabilities [11]: a uniform spatial density of the offered traffic in the range $0<r<1$, and thus

$$
f(r)= \begin{cases}2 r, & \text { for } 0<r<1 \\ 0, & \text { for } r>1\end{cases}
$$

is inserted in (12) and (15). In the Appendix it is shown that in this event, the capture probability, conditional on the number of interferers $i-1$, is the threefold integral

$$
\begin{gathered}
\operatorname{Pr}\{\text { capture } \mid i-1\}=\frac{2}{\sqrt{\pi}} \int_{0}^{1} \int_{-\infty}^{\infty} r_{t} \exp \left(-x^{2}\right) \\
\cdot\left[\frac{1}{\sqrt{\pi}} \int_{-\infty}^{\infty} f(x, y) \exp \left(-y^{2}\right) d y\right]^{i-1} d x d r_{t}
\end{gathered}
$$

with $f(x, y)$ defined by

$$
\begin{aligned}
f(x, y) \triangleq 1- & \left\{\sqrt{z} r_{t}^{2} \exp \left(\frac{1}{2} \sqrt{2} \sigma_{s}(y-x)\right)\right\} \\
\cdot & \arctan \left\{z^{-1 / 2} r_{t}^{-2} \exp \left(\frac{1}{2} \sqrt{2} \sigma_{s}(x-y)\right)\right\} .
\end{aligned}
$$

The probability $q_{i}$ that one out of $i$ packets captures the base 


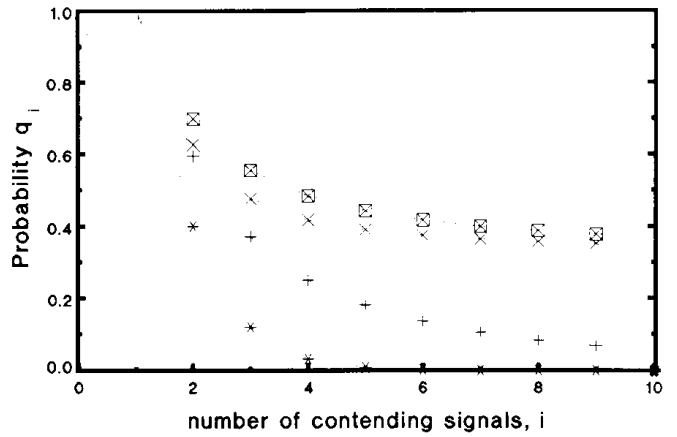

Fig. 3. Probability that one out of $i$ packets successfully captures the base station, for Rayleigh fading $(*)$, Rayleigh fading and shadowing $\left(\sigma_{s}=1.36\right.$ or $6 \mathrm{~dB})(+)$, near-far effect and Rayleigh fading $(x)$, and near-far effect, Rayleigh fading and shadowing $(\cdot)$. Receiver threshold $z=10$.

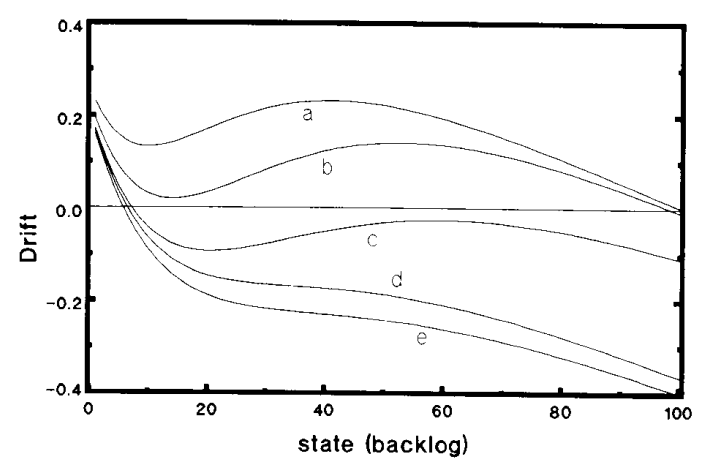

Fig. 4. Drift for a network of $N=100$ users for (a) a receiver without capture, and for a receiver with threshold of $6 \mathrm{~dB}(z=4)$ for a channel with (b) Rayleigh fading only, (c) shadowing $\left(\sigma_{s}=1.36\right.$ or $\left.6 \mathrm{~dB}\right)$ and Rayleigh fading, (d) near-far effect and Rayleigh fading (d), (e) near-far effect, shadowing $\left(\sigma_{s}=1.36\right)$ and Rayleigh fading.

station is found from

$$
q_{i}=i \operatorname{Pr}\{\text { capture } \mid i-1\}
$$

since the probability that two or more packets capture the receiver simultaneously is zero. Results are illustrated in Fig. 3 for various propagation models, assuming a receiver threshold of $z=4(6 \mathrm{~dB})$. The nonzero limit for $i \rightarrow \infty$ is due to the unrealistic traffic assumed in the immediate vicinity of the base station $(r \rightarrow 0)$.

\section{Network Stability}

Inserting the probabilities (18) and (17) in (1) and (2) gives the expected dynamic behavior of the network. In Fig. 4 , the dynamic behavior of the mobile network is shown for various propagation models in terms of the expected drift (2). A population of $N(N=100)$ terminals, spread with equal probability over the coverage area (16), and contending for a common receiver with a threshold of $z=4$, is assumed. The probability of packet generation is $p_{0}=0.0055$ and the probability of retransmission is $p_{r}=0.08$. If capture never occurs $\left(q_{i} \equiv 0\right.$ for $\left.i>1\right)$, entire saturation $\left(\pi_{s} \approx N=100\right.$ ) leads to extraordinarily high delay ( $D \approx 4.610^{5}$ slots). For the traffic parameters studied here, network performance is not essentially improved by Rayleigh fading only, e.g. if adaptive power control is applied to compensate slow power variations: steady-state operation is found at a backlog of nearly $\pi_{s} \approx 100$ terminals, leading to an average delay of $D \approx 7000$ slots. The curves $c, d, e$ in Fig. 4 for Rayleigh fading combined with shadowing or near-far effects all show a single, almost identical equilibrium with low average delay on the order of 10-15 time slots. Even if the near-far effect is ignored, i.e., $f(r)=\delta(r-1)$ in curve $c$, the network is stable and terminals experience little backlog. Therefore, it may be concluded that for the reported traffic parameters, the stability of the network does not rely on terminals unrealistically close to the base station.

The drift at high backlog, especially $d_{N}$, is mainly determined by the probability of capture in the event of many colliding terminals. Due to Rayleigh fading, the power level is usually relatively constant with occasional deep fades, whereas up-fades are rare. Hence, the probability that one out of many Rayleigh fading signals is sufficiently stronger than the joint interference is low as compared to other propagation mechanisms (see Fig. 3). Consequently, the drift from full saturation $d_{100}$ is almost zero in curve $b$. The log-normal distribution, due to shadowing, exhibits a higher probability of up-fades of the signal power, resulting in a negative drift of $d_{100} \approx-0.1$ away from the situation with all terminals in backlog. The spatial distribution (16), with finite traffic in the immediate vicinity of the receiver, has a fast recovery of about -0.4 backlogged terminals per time slot away from states with high backlog. In this event, $-d_{N}$ and $S_{N}$ roughly equal the capture probability $q_{\infty}$, since $q_{i}$ is almost constant for large $i$.

Fig. 5 gives the region for $p_{0}$ and $p_{r}$ where the network with $N=100$ terminals is bistable. The locus $(-)$ of the bifurcation set for ALOHA without capture $\left(q_{i} \equiv 0\right.$ for $i>0$ ) is taken from Onozato and Noguchi [14]. Recently, additional bifurcations sets were reported, mapping the stability of a slotted ALOHA network with capture where the users are divided into two groups [15]. A similar catastrophe theory for a full propagation model including Rayleigh fading, shadowing and the near-far effect, has not yet been developed. Our bifurcation set (- -) was estimated by trial and error with the technique used to obtain drift curves as in Fig. 4. The mobile network exhibits bistability at substantially higher packet traffic loads when receiver capture occurs, even for a receiver threshold of $10 \mathrm{~dB}(z=10)$. The region of bistability is relatively small: the transition through this area from a single (low-backlog) equilibrium into saturation occurs for relatively small increments of the packet generation probability $p_{0}$. In contrast to this, since the bifurcation set of the mobile net is almost parallel to $p_{r}$-axis, a change in the retransmission probability has less effect on the stability of the net. For a packet generation probability up to $p_{0}=$ 0.002 , even a persistent retransmission schedule $\left(p_{r}=1\right)$ leads to a stable network. Decreasing the generation probability $p_{0}$ always has a positive effect on the network performance, since the drift toward lower backlog increases for all $n$. Simulations [24] indicated that reducing the probability of retransmission $p_{r}$ has a positive effect on the network performance in saturated networks (increasing throughput, decreasing backlog, and delay), but a negative effect in stable, 


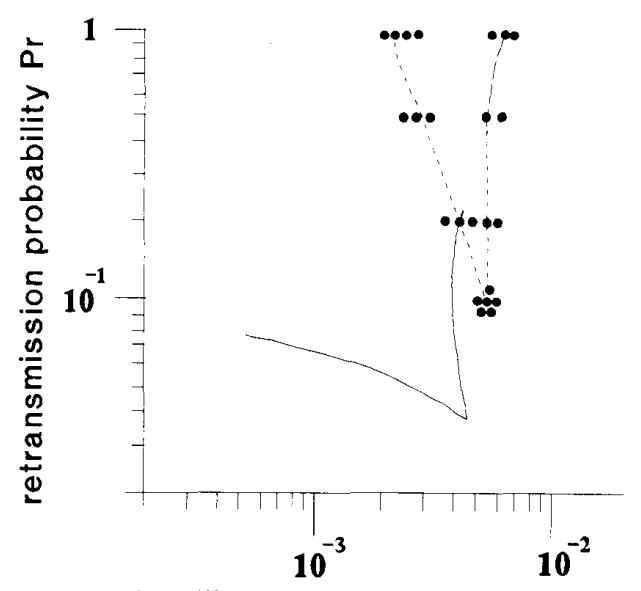

probability of packet generation Po

Fig. 5. Bistability area for a network with 100 users without capture $(-)$ [14] and with imperfect capture $(--)(z=10)$ of mobile signals. The latter bifurcation set is estimated by trial in 23 points $(\bullet)$.

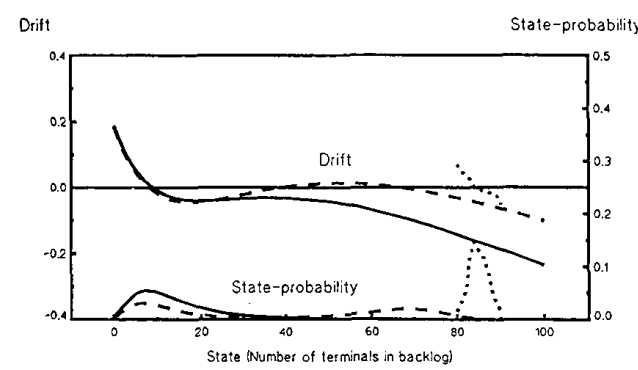

Fig. 6. Expected drift and state probability for a slotted ALOHA network with 100 users and a receiver threshold of $z=10$, according to a uniform distribution $(-)$, a log-normal spatial distribution with $\sigma_{d}=2(\approx 8.68$ $\mathrm{dB},--)$ and a simulation $(\cdots)$. The probability of generation of a new packet $p_{0}=0.0055$. The probability of retransmission is $p_{r}=0.08$.

unsaturated networks (lower throughput, increasing backlog, and delay). In bistable nets, appropriate reduction of $p_{r}$ can remove bistability. Simulation runs, however, indicated that the newly attained stable network usually is in relatively high backlog. By reducing the probability of retransmission, the effective time each terminal spends in the origination mode also reduces, which indirectly resulted in a low input traffic load. This suggests that mobile channels might as well be managed by directly controlling the input traffic, for instance by limiting the number of terminals $N$ that are allowed to be signed on simultaneously.

\section{NEAR-FAR EFFECT}

As in many other studies (e.g. [4], [11]), until now terminals were assumed to be (quasi-) uniformly distributed over the coverage area. As an alternative, the log-normal spatial distribution

$$
f(r)=\frac{\beta}{\sqrt{2 \pi} r \sigma_{d}} \exp \left\{-\frac{\beta^{2} \ln r^{2}}{2 \sigma_{d}^{2}}\right\},
$$

with $\sigma_{d}^{2}$ the spatial logarithmic variance, was suggested in [21]. This allows more realistic modeling of the packet traffic very close to the central receiver, since all (linear) moments of the pdf of received power are undefined for the uniform spatial density (16), whereas the log-normal distribution (19) gives finite moments [22]. Still, (16) provides a logarithmic standard deviation of the received power, viz. [21]

$$
\sigma_{d}^{2}=\beta^{2} \int_{0}^{1} \ln ^{2} r 2 r d r-\beta^{2}\left[\int_{0}^{1} \ln r 2 r d r\right]^{2}=\left(\frac{1}{2} \beta\right)^{2} \text {. }
$$

The true uniform distribution (16) should therefore be compared with a lognormal distribution with $\sigma_{d}=2(8.68 \mathrm{~dB})$, for $\beta=4$. The state probability $\pi_{n}$ and average drift $d_{n}$ are depicted in Fig. 6 for a receiver threshold of $z=10(10 \mathrm{~dB})$. For the selected traffic parameters $\left(p_{0}=0.0055, p_{r}=0.08\right.$ ), the two spatial models studied give different assessments of the stability of the network with $N=100$ terminals. While the assumption of a uniform (continuous) distribution of the offered traffic suggests a stable network with little backlog, the log-normal model predicts bistable performance.

Judging from the results of a simulation [24] which took into account the effect that most retransmissions originate from the boundary of the service area, both the uniform and 
the log-normal distributions lead to optimistic estimates of the network performance. Initially the software simulation program randomly distributes $N(N=100)$ terminals over the area $0<r<1$ and estimates the corresponding area-mean powers (7) for every terminal. All terminal states are modeled as a tristate variable. In the $O$ state, the program performs a random experiment to simulate generation of a packet with probability $p_{0}$. In the $R$ state, permission for retransmission of a previously collided packet is granted with probability $p_{r}$. In both cases, the terminal enters the $T$ state where the transmission and propagation of a packet is simulated by the random generation of an instantaneous received power $p_{s}$, according to an exponential distribution with mean (1). Shadowing is ignored in this experiment (i.e., $\sigma_{s}=0$ ). By accounting for all the received interference packets, the program determines whether any of the colliding packets is strong enough to capture the receiver.

Due to the near-far effect, the average time a terminal is in the retransmission mode increases with distance. Since $p_{0}<p_{r}$, the traffic offered per unit of area increases with distance. This has a disadvantageous influence on the network performance: colliding signals are more often received from far away [3], [8], [9] and hence with almost equal (low) powers. Consequently, the probability $q_{i}$ that the power of one of the signals largely exceeds the joint interference is relatively low.

\section{Conclusion}

A weight function approach has been developed to investigate the combined influence of three types of mobile fading on capture probabilities in the mobile packet radio. It has been observed that inclusion of Rayleigh fading actually facilitates mathematical analysis, rather than complicating derivations. To obtain capture probabilities in the presence of simultaneous Rayleigh fading, shadowing and near-far effect, an integral solution using a numerical method (the Hermite polynomial method) is possible. Consequently, Monte Carlo simulation, as used e.g. in [11], or approximate methods matching only the first- and second-order moments of the pdf's [22], [7], [10], can be avoided. Results have been presented for a uniform spatial distribution of the packet traffic offered to the ALOHA channel, and the influence of alternative modeling of the near-far effect has been investigated.

Our calculations reveal that bistability of the mobile networks considered occurs only for a very limited range of transmission parameters. The transition from low-backlog to saturation, i.e., the transition through the area of bistability, is very abrupt. This suggests that, in contrast to the case for wired networks, protocol design should focus on continuously assuring sufficient throughput and acceptable delay, for instance of exercising (slow) centralized control over the (number of) terminals allowed to sign on. The effects of the various propagation mechanisms on network performance is clearly exhibited at high backlog. The probability that an individual signal is sufficiently strong to survive a collision with many contenders is very small in channels with Rayleigh fading only. This type of channels fails to recover rapidly from situations of high backlog. Shadowing enhances the drift toward a lower backlog. The near-far effect gives nearby users a further increased probability to survive a collision. This effect was found particularly effective in retrieving the network from high backlog.

In mobile ALOHA networks, issues of (dynamically) resolving instability and collision resolution are presumably less critical compared with networks without capture. However, the near-far effect may not always be modelled by a (continuous) uniform spatial density of the traffic. This popular model fails in two respects.

1) It allows terminals to be infinitely close to the base station. For high traffic loads, stability may therefore rely upon terminals unrealistically close to the base station;

2) Another effect is the interaction between the attempted traffic and the system performance: almost all retransmissions are clustered in (distant) parts of the coverage area with poor propagation. Since these transmissions may arrive with almost equal (and low) power, mutual collisions usually result in the loss of all packets involved. Throughput and delay are thus less enhanced by capture than suggested by the theoretical investigations of (quasi-) uniform spatial distributions reported recently. Similarly, the assessment of stability areas in this paper may be further refined by investigating the drift at high backlog from a nonuniform and dynamically changing spatial distribution of the backlogged terminals.

A similar effect as in 2) is believed to occur in networks with stationary terminals, such as in narrow-band indoor wireless office communication. The main cause of fading, transmitter mobility, is not present for terminals at a fixed location. Transmitters accidentally placed near a multipath null generate the main part of all retransmissions. Simulation revealed that area-mean powers remaining fixed for each terminal due to virtually constant propagation distances degrades the performance for mobile networks. Presumably, constant shadow and multipath attenuation during retransmissions in fixed networks further degrades overall performance. A more detailed study of the network performance for stationary subscribers is recommended.

\section{APPENDIX}

Capture Probabilities for Special Cases of the Spatial Distribution

\section{A. Uniform Spatial Distribution}

For a uniform distribution of the offered traffic (16), the Laplace image (11) of the (unconditional) pdf of received power $P_{s}$ is

$$
\begin{aligned}
\phi_{P s}\left(\frac{z}{p_{1 t}}\right)= & \int_{0}^{1} \frac{2 r}{\sqrt{\pi}} \\
& \cdot \int_{-\infty}^{\infty} \frac{p_{1 t} \cdot r^{\beta} \cdot \exp \left(-y^{2}\right)}{p_{1 t} \cdot r^{\beta}+z \cdot \exp \left(y \sigma_{s} \sqrt{2}\right)} d y d r
\end{aligned}
$$

where

$$
y^{2} \triangleq \frac{\ln ^{2}\left(p_{1} \cdot r^{\beta}\right)}{2 \sigma_{s}^{2}}
$$


has been substituted. After substitution of

$$
t \triangleq \frac{r^{\beta} p_{1 t}}{z \exp \left(y \sigma_{s} \sqrt{2}\right)},
$$

the image for UHF groundwave propagation $(\beta=4)$ becomes

$$
\begin{aligned}
\phi_{P s}\left(\frac{z}{p_{1 t}}\right)= & \frac{1}{2 \sqrt{\pi}} \int_{-\infty}^{\infty} \int_{0}^{t_{\max }} \frac{\sqrt{t}}{1+t} d t \\
& \cdot \frac{\sqrt{z}}{\sqrt{p_{1 t}}} \exp \left\{\frac{1}{2} \sqrt{2} y \sigma_{s}\right\} \exp \left\{-y^{2}\right\} d y
\end{aligned}
$$

with $t_{\max }$ defined as

$$
t_{\max } \triangleq \frac{p_{1 t}}{z} \exp \left(-y \sigma_{s} \sqrt{2}\right) .
$$

Using $[23,14.115]$, the image function becomes

$$
\begin{aligned}
& \phi_{P s}\left(\frac{z}{p_{1 t}}\right)=\frac{1}{\sqrt{\pi}} \int_{-\infty}^{\infty}\left[1-\frac{\sqrt{z}}{\sqrt{p_{1 t}}} \exp \left\{\frac{1}{2} \sqrt{2} y \sigma_{s}\right\}\right. \\
& \left.\cdot \arctan \left(\frac{\sqrt{p_{1 t}}}{\sqrt{z}} \exp \left\{-\frac{1}{2} \sqrt{2} y \sigma_{s}\right\}\right)\right] \exp \left\{-y^{2}\right\} d y
\end{aligned}
$$

which is to be inserted in (15). The integral over $p_{1 /}$ is rewritten, using the substitution

$$
x^{2}=\frac{\ln ^{2}\left(p_{1 t} r_{t}^{\beta}\right)}{2 \sigma_{s}^{2}} .
$$

The probability of capture, conditional on the number of interferers $i-1$, is the three-dimensional integer

$$
\begin{gathered}
\operatorname{Pr}(\text { capture } \mid i-1)=\frac{2}{\sqrt{\pi}} \int_{0}^{1} \int_{-\infty}^{\infty} r_{t} \exp \left(-x^{2}\right) \\
\cdot\left[\frac{1}{\sqrt{\pi}} \int_{-\infty}^{\infty} f(x, y) \exp \left(-y^{2}\right) d y\right]^{i-1} d x d r_{t}
\end{gathered}
$$

with $f(x, y)$ defined by

$$
\begin{aligned}
f(x, y) & \triangleq 1-\sqrt{z} r_{t}^{2} \exp \left\{\frac{1}{2} \sqrt{2} \sigma_{s}(y-x)\right\} \\
\cdot & \arctan \left\{z^{-1 / 2} r_{t}^{-2} \exp \left\{\frac{1}{2} \sqrt{2} \sigma_{s}(x-y)\right\}\right\} .
\end{aligned}
$$

\section{B. Log-Normal Spatial Distribution}

For the log-normal distribution of the area-mean power (19), the capture probability becomes

$$
\begin{aligned}
q_{i}= & i \int_{0}^{\infty} d y_{0} \frac{1}{\sqrt{\pi}} \exp \left\{-y_{0}^{2}\right\} \\
& \cdot\left[\frac{1}{\sqrt{\pi}} \int_{-\infty}^{\infty} \frac{\exp \left\{y_{0} \sigma \sqrt{2}\right\} \exp \left\{-y^{2}\right\}}{\exp \left\{y_{0} \sigma \sqrt{2}\right\}+z \exp \{y \sigma \sqrt{2}\}} d y\right]^{i-1}
\end{aligned}
$$

with $\sigma^{2} \triangleq \sigma_{s}^{2}+\sigma_{d}^{2}$. In [10], the steady-state throughput of a mobile ALOHA network with Rayleigh fading and shadowing was estimated from an approximation technique for assessing the log-normal distribution of the joint interference signal [22]. It has been verified that the exact formula (30) gives almost identical results.

For the uniform and log-normal spatial distribution, numerical results for the capture probability (28)-(30) have been obtained using the Hermite polynomial method. Both infinite integrals in (28) and (30) are approximated using

$$
\int_{-\infty}^{\infty} f(y) \exp \left\{-y^{2}\right\}=\sum_{m=1}^{M} w_{m} f\left(y_{m}\right)+R_{M}
$$

For an $M$-point integration, the weight factors $w_{m}$ in the sample points $y_{m}$ are in [19, table 25.10]. The remainder $R_{M}$ is

$$
R_{M}=\frac{M ! \sqrt{\pi}}{2^{M}(2 M) !} f^{(2 M)}(\xi)
$$

with $\xi$ a point in the interval $(-\infty, \infty)$. For integrands in (29) and (30), the $2 M$ th derivative can be shown to be bounded to some constant, e.g. $f^{(2 M)}(\xi)<C_{M}$ for all $\xi$. Since $C_{M}$ does not increase substantially with $M$, the remainder $R_{M}$ rapidly vanishes for increasing $M$. It appeared that, for $M$ larger than say 10 or 20 , the numerical results becomes almost independent of the number of samples $M$. More critical is the evaluation of the integral over $r_{t}$ in (28) and (29), since the number of samples near the limit $r_{t}=0$ may need to be increased for a large number of contending signals (large $i$ ). This is because the main contribution of the capture probability occurs for signals transmitted very close to the base station (small $r_{t}$ ). It is our experience that in particular after substituting the distance of the test packet into any other integration variable, the accuracy of the numerical method should be examined carefully.

\section{REFERENCFS}

[1] N. Abramson, "The ALOHA system-Another alternative for computer communications," in Proc. 1970 Fall Joint Comput. Conf. AFIPS Press, vol. 37,1970 , pp. 281-285.

[2] L. Kleinrock, Queueing systems, vol. 1: Theory, vol. 2: Computer Applications. New York: Wiley, 1975, 1976.

[3] N. Abramson, "The throughput of packet broadcasting channels," IEEE Trans. Commun., vol. COM-25, pp. 117-128, Jan. 1977.

[4] J. C. Arnbak and W. van Blitterswijk, "Capacity of slotted-ALOHA in a Rayleigh fading channel," IEEE J. Select. Areas Commun. vol. SAC-5, pp. 261-269, Feb. 1987.

[5] I. M. I. Habbab, M. Kavehrad, and C-E. W. Sundberg, "ALOHA with capture over slow and fast fading radio channels with coding and diversity," IEEE J. Select. Areas Commun., vol. 7, pp. 79-88, Jan. 1989.

[6] D. J. Goodman and A. A. M. Saleh, "The near/far effect in local ALOHA radio communications," IEEE Trans. Veh. Technol, vol. VT-36, pp. 19-27, Feb. 1987

[7] R. Prasad and J. C. Ambak, "Enhanced throughput in packet radio channels with shadowing," Electron. Lett., vol. 24, pp. 986-988, Aug. 4, 1988.

[8] J. P. M. G. Linnartz, R. Prasad, and J. C. Arnbak, "Spatial distribution of traffic in a cellular ALOHA network," $A E U$, vol. 42 , no. 1, pp. 61-63, Jan./Feb. 1988.

[9] J. P. M. G. Linnartz and J. C. Arnbak, "Spatial distribution of traffic in a mobile ALOHA network," in Conf. Proc. Area Commun., EUROCON 88, Stockholm, June 1988, pp. 326-329.

[10] R. Prasad and J. C. Arnbak, "Effects of Rayleigh fading on packet 
radio channels with shadowing," in Proc. IEEE Tencon 1989, Bombay, India, Nov. 1989 , pp. 546-548.

[11] C. Namislo, "Analysis of mobile radio slotted ALOHA networks,' IEEE J. Select. Areas Commun., vol. SAC-2, pp. 583-588, July 1984

[12] A. B. Carleial and M. E. Hellman, "Bistable behavior of ALOHAtype systems,"' IEEE Trans. Commun., vol. COM-23, pp. 401-410, Apr. 1975

[13] L. Kleinrock and S. S. Lam, "Packet switching in a multiaccess broadcast channel-performance evaluation," IEEE Trans. Commun., vol. COM-23, pp. 410-423, Apr. 1975.

[14] Y. Onozato and S. Noguchi, "On the thrashing cusp in slotted ALOHA systems," IEEE Trans. Commun., vol. COM-33, pp. 1171-1182, Nov. 1985

[15] Y. Onozato, J. Liu, and S. Noguchi, "Stability of a slotted ALOHA system with capture effect," IEEE Trans. Veh. Technol., vol. 38, pp. 31-36, Feb. 1989.

[16] W. C. Y. Lee, Mobile Communications Design Fundamentals. Indianapolis, IN: Howard W. Sams, 1986.

[17] F. Hansen en F. Meno, "Mobile fading-Rayleigh and lognormal superimposed," IEEE Trans. Veh. Technol., vol. VT-26, pp. 332-335, Nov. 1977

[18] J. P. M. G. Linnartz and R. Prasad, "Threshold crossing rate and average non-fade duration in a Rayleigh-fading channel with multiple interferers," $A E \ddot{U}$, vol. 43, no. 6, pp. 345-349, Nov./Dec. 1989.

[19] Handbook of Mathematical Functions. M. Abramowitz and I. A Stegun, Eds. New York: Dover, 1965.

[20] D. Verhulst, M. Mouly, and J. Szpirglas, "Slow frequency hopping multiple access for digital cellular radiotelephone," IEEE J. Select. Areas Commun., vol. SAC-2, pp. 563-574, 1984.

[21] J. P. M. G. Linnartz and R. Prasad, "Near-far effect on slotted ALOHA channels with shadowing and capture," in Proc. IEEE Veh. Technol. Conf. 1989, San Francisco, May 3-5, pp. 809-813.

[22] S. C. Schwartz and Y. S. Yeh, "On the distribution function and moments of power sums with log-normal components," Bell Syst. Tech. J., vol. 24 , pp. $316-317,1982$

[23] M. R. Spiegel, Mathematical Handbook of Formulas and Tables. New York: McGraw-Hill, Schaum's Outline Series, 1968.
[24] C. van der Plas, "Stability of mobile slotted ALOHA networks," M.Sc. E.E. thesis, Delft Univ. Technol., the Netherlands, no. 168250-289, Sept. 1989.

[25] C. Lau and C. Leung, "Capture models for mobile packet radio networks," in Proc. Int. Conf. Commun., ICC, Atlanta, Apr. 1990, pp. 1226-1230.

[26] S. Ghez, S. Verdú, and S. C. Schwartz, "Stability properties of slotted ALOHA with multipacket reception capability," IEEE Trans. Automat. Contr., vol. 33, pp. 640-649, July 1988.

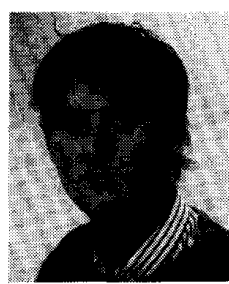

Cornelis van der Plas was born in Katwijk, the Netherlands, in 1966. He received the Ir. (M.Sc.E.E.) degree in electrical engineering from Delft University of Technology, the Netherlands, in 1989 .

His dissertation was on stability of mobile slotted ALOHA networks. After his graduation he joined Intercai B.V., a consultancy company for telecommunications. After completing his training in a consultancy course, he now works as a consultant for telecommunications.

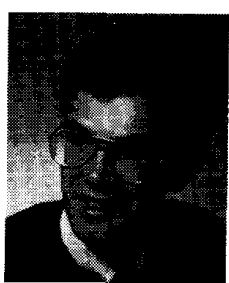

Jean-Paul M. G. Linnartz (' ' $86-\mathbf{M}^{\prime} 90$ ) was born in Heerlen, the Netherlands, in 1961 . He received the Ir. (M.Sc.E.E.) degree in electrical engineering with distinction from Eindhoven University of Technology, the Netherlands, in 1986.

During 1987-1988 he was with Physics and Electronics Laboratory F.E.L.-T.N.O., the Hague where he was involved in UHF propagation and frequency assignment. Since 1988 he has been an assistant professor at Delft University of Technology, where he is working on multiuser land-mobile radio nets. He is also interested in radio broadcasting. 